\title{
Lutein and Zeaxanthin Supplementation in Preterm Very Low-Birth-Weight Neonates in Neonatal Intensive Care Units: A Multicenter Randomized Controlled Trial
}

\author{
Paolo Manzoni, MD ${ }^{1}$ Roberta Guardione, RN ${ }^{1}$ Paolo Bonetti, MD ${ }^{2}$ Claudio Priolo, MD ${ }^{1}$ \\ Andrea Maestri, MD ${ }^{1}$ Caterina Mansoldo, MD ${ }^{2}$ Michael Mostert, MD 3, 5 \\ Giovanni Anselmetti, MD ${ }^{4}$ Daniela Sardei, MD ${ }^{3,5}$ Massimo Bellettato, MD 3,5 \\ Paolo Biban, MD $^{2}$ Daniele Farina, MD $^{1}$
}

\footnotetext{
${ }^{1}$ Neonatology and NICU, S. Anna Hospital, Torino, Italy

2 Pediatrics/Neonatal Intensive Care Unit, Division of Pediatrics, Azienda Ospedaliera, Verona, Italy

${ }^{3}$ Division of Neonatology and Pediatrics, City Hospital, Thiene, Italy

${ }^{4}$ Referral Center for ROP of Piedmont, Torino, Italy

${ }^{5}$ Department of Pediatrics, University of Torino, Italy
}

\begin{abstract}
Address for correspondence and reprint requests Paolo Manzoni, MD, Neonatology and NICU, S. Anna Hospital, Azienda Ospedaliera, Regina Margherita, S. Anna, C. Spezia 60, 10126 Torino, Italy (e-mail: paolomanzoni@hotmail.com).
\end{abstract}

\begin{abstract}
Keywords

- nutrition

- lutein

- zeaxanthin

- preterm neonates

- ROP

$-B P D$

- NEC

- prevention

Background Human milk feeding protects against oxidative stress-induced damage in preterm neonates, including severe multifactorial diseases such as retinopathy of prematurity (ROP), necrotizing enterocolitis (NEC), and bronchopulmonary dysplasia (BPD). The carotenoids, which are not found in formula milk, might play a key role in these actions.

Methods A multicenter, double-blind, randomized controlled trial was conducted in three tertiary Italian neonatal intensive care units. All preterm infants $<32^{+6}$ weeks' gestational age were eligible and were randomized to a single, oral, daily $0.5-\mathrm{mL}$ dose of carotenoid supplementation $(0.14 \mathrm{mg}$ lutein $+0.0006 \mathrm{mg}$ zeaxanthin) or placebo ( $5 \%$ glucose solution) from birth till 36 weeks' corrected gestational age. Primary outcomes were threshold ROP, NEC > second stage, and BPD. Surveillance for detection of these diseases and for intolerance/adverse effects was performed.

Results No treatment-related adverse effect was documented in the 229 analyzed infants, whose clinical/demographical characteristics were similar in the two groups. Threshold ROP incidence did not significantly differ in treated (6.2\%) versus not treated infants $(10.3 \% ; p=0.18)$. The same occurred for NEC $(1.7 \%$ versus $5.1 \% ; p=0.15)$ and BPD ( $4.5 \%$ versus $10.3 \%$; $p=0.07)$. Noteworthy, the progression rate from early ROP stages to threshold ROP was decreased by $50 \%$ ( 0.30 versus $0.44 ; p=0.23$ ).

Conclusion Lutein/zeaxanthin supplementation in preterm infants is well tolerated. No significant effect was seen on threshold ROP, NEC, or BPD. The decreasing trends of these outcomes in the treatment group need to be assessed and confirmed on larger sample-sizes.
\end{abstract}

received

November 14, 2011

accepted after revision

March 16, 2012

published online

July 6, 2012
Copyright $\odot 2013$ by Thieme Medical Publishers, Inc., 333 Seventh Avenue, New York, NY 10001, USA. Tel: +1(212) 584-4662.
DOI http://dx.doi.org/ 10.1055/s-0032-1321494. ISSN 0735-1631. 
Progress in perinatal care has improved survival rates among preterm neonates. However, disability-free survival from preterm birth is increasingly hampered by several adverse, long-term outcomes of prematurity such as retinopathy of prematurity (ROP), necrotizing enterocolitis (NEC) and bronchopulmonary dysplasia (BPD). ${ }^{1}$

Prematurity itself plays the major role in such multifactorial late diseases. However, oxidative stress-induced damage related both to prematurity and to the type of care administered is speculated to play an important, additional role. ${ }^{2}$ As these preterm infants are susceptible to oxidative damage due to high metabolic rate and low levels of antioxidant enzymes, and this is a crucial step for the onset of many severe outcomes of prematurity, efforts have been addressed to the identification of protective strategies that will enhance their antioxidant functions. ${ }^{3}$

Maternal milk feeding is indicated for all premature infants as it provides valuable nutritional and immunologic benefits. ${ }^{4}$ Noteworthy, human milk is also protective toward some severe outcomes of prematurity such as sepsis, ${ }^{4}$ ROP, $\mathrm{BPD}$, and NEC. 6

The observation that fresh human milk is rich in many antioxidants $^{7}$ (e.g., vitamin E, $\beta$-carotene, lutein, and lactoferrin) helps understand why human milk has is protective against many multifactorial diseases in which an oxidative stress-induced damage occurs (e.g., ROP, NEC, and BPD).

Among the antioxidant factors of human milk, the carotenoids (lutein, $\beta$-carotene, zeaxanthin, lycopene) are believed to play a crucial role. ${ }^{8}$ Carotenoids, a family of polyene lipophilic molecules, are not found in formulas. Colostrum contains a very high level of carotenoids, particularly of lutein (up to $140 \mu \mathrm{g} / \mathrm{L}$ ) that is fivefold that of mature milk. ${ }^{9}$ Carotenoids provide protection against both lightinduced and metabolic oxidative damage in the premature retina, and protection from oxidative stress in other developing tissues where oxidative insults occur.

As nutritional supplements, lutein and zeaxanthin were granted a generally recognized as safe (GRAS) status by the Food and Drug Administration in June 2004 (see GRAS Notice No. GNR 000140 issued by Center for Food Safety and Applied Nutrition/Office of Food Additive Safety ${ }^{10}$ ). On this ground, these two carotenoids are currently marketed worldwide, but only in some countries is luteinsupplemented formula milk available.

The present study assesses the outcomes of preterm infants given supplemented carotenoids (lutein and zeaxanthin) during their stay in the neonatal intensive care unit (NICU), as well as the safety and tolerability of these two supplements.

\section{Methods}

This study is a multicenter, prospective, randomized, doubleblind, placebo-controlled trial conducted over a 12-month period in three NICUs of northern Italy.

All neonates with gestational age $<32$ weeks +6 days (i.e., all those qualifying for screening of ROP) born within the study period, whether at one of the participant institutions or elsewhere, were eligible for the study. Parental refusal, admission after 48 hours of life, death prior to 72 hours of life, ophthalmologic disease already present at the time of randomization were exclusion criteria.

The primary objective of the study was to evaluate the effectiveness of the lutein + zeaxanthin supplementation, compared with placebo, in the prevention of threshold ROP, $\mathrm{BPD}$, and necrotizing enterocolitis (NEC) of surgical stage (i.e., second or greater according to Bell's classification ${ }^{11}$ ).

Secondary objectives were the assessment of the incidence of ROP of all stages, NEC of all stages; of intestinal perforation; of late-onset sepsis; of mortality prior to discharge; of severe (grade 3 to 4 ) intraventricular hemorrhage; of need for transfusions; of liver failure defined as $3 \times$ elevation of normal serum liver enzymes values.

Surveillance for detection of all these outcomes, as well as for episodes of intolerance to the supplements or adverse effects, was performed till discharge or term corrected age, whichever came first. Measurements of serum liver enzymes values were performed at 4 weeks of age.

Infants were randomly allocated to one of two groups in a 1:1 ratio to receive carotenoids oral supplementation (group A) or placebo (group B). Randomization was stratified by center, and randomly permuted blocks of size 9 and 12 were used. The random allocation sequence was generated using ralloc.ado version 3.2.5 in Stata 9.2 (Stata-Corp, College Station, TX). The pharmacy at each center used these computer-generated randomization lists to form the two groups and prepared the drug doses in sealed opaque vials.

In detail, the study intervention consisted of daily oral administration of a mixture containing $0.14 \mathrm{mg}$ of lutein and $0.0006 \mathrm{mg}$ of zeaxanthin (equal to $0.5 \mathrm{~mL}$ of the product LuteinOfta gtt, NEOOX Division of SOOFT Italia SpA, Montegiorgio, Italy) in group A infants, whereas group B infants received a daily oral administration of $0.5 \mathrm{~mL}$ of $5 \%$ glucose solution as placebo. Clinical and research staff remained unaware of study group assignments during the study.

As mentioned, the supplemented drug and placebo were administered in a single oral daily dose from birth till week 36 of gestational (corrected) age, starting from the first 48 hours of life.

Neonates not feeding in the first 48 hours received the drug/placebo by oral/nasogastric tube and were enrolled in the absence of gastric instability and/or repeated gastric residuals or emesis. If they repeatedly displayed gastric instability, gastric residuals, or vomit, they were enrolled at any point during the first week of life, depending on the first "efficacious" feedings. The day of life in which they first received the drugs or placebo was recorded in the database, and their statistics were limited to the days of administration/ exposure to the intervention.

Nutritional and feeding policies followed common protocols. Administration of fresh, expressed maternal milk was encouraged. Each mother could supply milk only for her infant. When needed, feeding was supplemented with a formula for very low-birth-weight (VLBW) infants (PreAptamil; Milupa Italia, Milano, Italy) not supplemented with lutein or zeaxanthin. Systematic surveillance of adverse events (e.g., vomiting, feeding intolerance, skin rashes) was 
performed through daily infant examination until 2 days after end of treatment.

SOOFT Italia SpA supplied the lutein + zeaxanthin preparations, as well as the placebo in identical vials.

\section{Definitions of Outcomes}

NEC was defined as clinical signs with the presence of Pneumatosis intestinalis on abdominal X-rays, according to the Bell criteria. ${ }^{11}$

Severe BPD was defined as use of supplemental oxygen for 28 days plus $30 \%$ oxygen, positive pressure ventilation at 36 weeks' postmenstrual age, or both. ${ }^{12}$

ROP was defined according to the Early Treatment for Retinopathy of prematurity study. ${ }^{13}$ Ophthalmologic screening for ROP was performed by one board-certified consultant ophthalmologist in each participating unit. Infants were first screened at 3 to 4 weeks of age and then at 1- to 2-week intervals, depending on the clinical picture and the severity of retinopathy at the discretion of the ophthalmologist and neonatologist. All infants were examined until regression of the ROP lesions, or until retinal vascularization was complete. In case of discharge prior to the week 36 of gestational age, infants with ongoing not threshold ROP lesions were considered still at risk of progression to more severe stages, and therefore the screening was not discontinued. The infants were revisited also after discharge by the same ophthalmologists in the hospital unit, at scheduled intervals, until either the development of ROP or the disappearance of the lesions. Gestational age was calculated using the expected date of delivery based on an ultrasound performed before 22 weeks' gestation or-when ultrasound was not available-was determined by the attending neonatologist when the infant was admitted to the NICU based on neonatal clinical findings.

Neonates were all classified by their most severe ROP examination.

The screening ophthalmologists were unaware of treatment assignments or any other potential risk factors for ROP other than VLBW or gestational age $\leq 32$ weeks. The decision to treat ROP was always taken according to the stage of the disease.

Late-onset sepsis was defined as occurring more than 72 hours after birth and before discharge. This condition was based on the detection of clinical signs and symptoms by the physician in charge, presence of laboratory findings consistent with sepsis, and isolation of a causative organism from blood (drawn from peripheral sites) or cerebrospinal or peritoneal fluid. ${ }^{14,15}$ Diagnostic criteria were based on the existing literature, guidelines from international consensus documents, and recommendations from the Italian Neonatology Society's Fungal Infections Task Force. ${ }^{16,17}$

Presence and grade of intraventricular hemorrhage were documented by the most negative ultrasound finding available; intraventricular hemorrhage was classified by the Papile criteria. $^{18}$

\section{Statistical Analysis}

All primary and secondary outcomes were represented by dichotomous variables (presence/absence) and analyzed by intention-to-treat.
Categorical predictor variables were represented by percentages. Birth weight, gestational age, Apgar score, number of days receiving a given treatment, and daily amount of milk intake were represented by continuous variables. A complete list of the categorical and continuous variables considered is shown in - Table 1.

Proportions and continuous variables were compared using the Fisher exact two-tailed test and the $t$ test, respectively. Risk ratios and 95\% confidence intervals were calculated to compare cumulative between group incidences using Stata version 9.2.

The Wald test was used to assess the significance of the estimated coefficients. The likelihood ratio test was used to test the significance of the center-level variance component. Goodness-of-fit was evaluated through the log-likelihood of the fitted model. All tests were two-tailed, and $p<0.05$ was considered statistically significant.

Sample size analysis predicted that 114 patients would be needed for each group, based on two-sided type I error rates of 0.05 or less and $80 \%$ power to detect a relative difference between treated and nontreated infants of at least $66 \%$ (decrease from 18 to $6 \%$, given a pretrial incidence of $18 \%{ }^{15}$ ) for threshold ROP.

A total of 638 and 376 infants in each group would have been needed to detect the same extent of significant differences between groups for BPD and NEC, given pretrial incidences of $3.6 \%$ and $6.0 \%$, respectively. Given the low incidence of these last two outcomes in our pretrial data, the study was underpowered to detect possible significant differences.

Power calculations were performed according to $S$ plus, Version 2000. Analysis of dichotomous outcomes and interpretation of results were performed as suggested in Cochrane Reviewers' Handbook 4.2.2. ${ }^{19}$

SOOFT Italia SpA provided financial support with a grant but was not involved in the concept, design, enrollment, data collection, analysis and interpretation of its results, and decisions inherent the publication of the results.

\section{Results}

Of 247 VLBW infants considered for inclusion in the trial (-Fig. 1), 18 were not eligible either because they did not meet inclusion criteria $(n=1)$ or because the parents refused to participate $(n=14)$, or for other reasons $(n=3)$.

One infant in group A did not receive all the study doses, and four infants (two in group A and two in group B) had incomplete data, but all of them were included in the final analysis on an intent-to-treat basis.

The final analysis included 229 infants, 113 in group A and 116 in B. Clinical and demographic characteristics did not differ between the two groups (-Table $\mathbf{1}$ ).

Results of the study outcomes are reported in - Table 2. Overall, threshold ROP incidence tended to be lower in the treated (6.2\%) versus not treated (10.3\%) infants $(p=0.18)$. The same occurred for BPD ( $4.5 \%$ versus $10.3 \% ; p=0.15$ ) and NEC (1.7 versus $5.1 \% ; p=0.07$ ). Of note, treatment was associated with a lower rate of progression from the early stages of ROP to the threshold stage ( 0.30 versus 0.44 ; $p=0.23$ ). 
Table 1 Demographics and Clinical Characteristics

\begin{tabular}{|c|c|c|c|}
\hline & $\begin{array}{l}\text { Group A: Lutein } \\
(n=113)\end{array}$ & $\begin{array}{l}\text { Group B: Placebo } \\
(n=116)\end{array}$ & $\begin{array}{l}p \text { Value } \\
\text { Group A versus } \\
\text { Group B }\end{array}$ \\
\hline \multicolumn{4}{|l|}{ Demographics } \\
\hline Birth weight $(g)$, mean \pm SD (range) & $\begin{array}{l}1336 \pm 417 \\
(560-1485)\end{array}$ & $\begin{array}{l}1271 \pm 386 \\
(600-1500)\end{array}$ & 0.29 \\
\hline Gestational age (wk), mean \pm SD (range) & $\begin{array}{l}30.1 \pm 1.8 \\
(24-34)\end{array}$ & $\begin{array}{l}29.7 \pm 2.6 \\
(25-34)\end{array}$ & 0.45 \\
\hline Sex (male/female) & 44 & 56 & \\
\hline Race (\% of Caucasian) & $85 \%$ & $89 \%$ & 0.90 \\
\hline Born at another facility (\%) & 16 & 21 & 0.39 \\
\hline Vaginal delivery (\%) & 22 & 29 & 0.40 \\
\hline Mother had preeclampsia (\%) & 21 & 26 & 0.55 \\
\hline Premature rupture of membranes & 23 & 25 & 0.99 \\
\hline Use of antenatal corticosteroids & 63 & 64 & 0.89 \\
\hline Use of antenatal antibiotics & 70 & 73 & 0.99 \\
\hline \multicolumn{4}{|l|}{ Clinical characteristics } \\
\hline Mean Apgar score at 5 min. & 7.3 & 7.3 & 0.69 \\
\hline Use of surfactant (at least once) & 61 & 68 & 0.26 \\
\hline Umbilical catheter positioned (d) & 4.3 & 4.7 & 0.90 \\
\hline Intubation (d) & 5.6 & 7.0 & 0.42 \\
\hline Mechanical ventilation (total d) & 7.9 & 10.8 & 0.06 \\
\hline Supplemental oxygen (total d) & 9.6 & 12.9 & 0.25 \\
\hline Incidence of early-onset neutropenia (\%) & 8.5 & 9 & 0.80 \\
\hline Use of TPN (d) & 9.8 & 13.6 & 0.05 \\
\hline H2 blockers (total d) & 5.3 & 6.4 & 0.40 \\
\hline Third-generation cephalosporins (total d) & 3.5 & 4.1 & 0.88 \\
\hline Antibiotics (total d) & 12.6 & 15.4 & 0.19 \\
\hline Postnatal steroids (total d) & 0.4 & 1.2 & 0.07 \\
\hline Mean duration of stay in NICU (d) & 42 & 45 & 0.99 \\
\hline Central venous catheter(s) positioned (d) & 16.4 & 20.1 & 0.10 \\
\hline \multicolumn{4}{|l|}{ Nutritional characteristics } \\
\hline Time of initiation of oral feeding (DOL) & 3.3 & 3.8 & 0.14 \\
\hline Time of achievement of full feeding (DOL) & 12.7 & 14.3 & 0.25 \\
\hline Mean volume of feedings advancements daily $(\mathrm{mL} / \mathrm{d})$ & 10.0 & 10.5 & 0.69 \\
\hline Proportions of infants fed with only formula & $19 \%$ & $17 \%$ & 0.70 \\
\hline Daily average amounts of human fresh milk intake $(\mathrm{mL} / \mathrm{kg})$ & 68.5 & 65.0 & 0.99 \\
\hline Total days of human fresh milk feeding & 30 & 29 & 0.99 \\
\hline
\end{tabular}

DOL, day of life; NICU, neonatal intensive care unit; SD, standard deviation; TPN, total parenteral nutrition.

No significant differences were seen also when clustering the analysis for type of infant feeding (human fresh milk versus formula milk).

Serum liver enzymes values at 4 weeks of age were normal and comparable in the two groups (-Table 3 ). Liver function evaluations did not disclose any hepatic adverse effect putatively attributable to the supplementation in any moments.
No adverse effects putatively attributable to the treatment was documented.

\section{Discussion}

To our knowledge, this is the first randomized controlled trial (RCT) assessing the outcomes of preterm infants given a carotenoid supplementation since birth. 


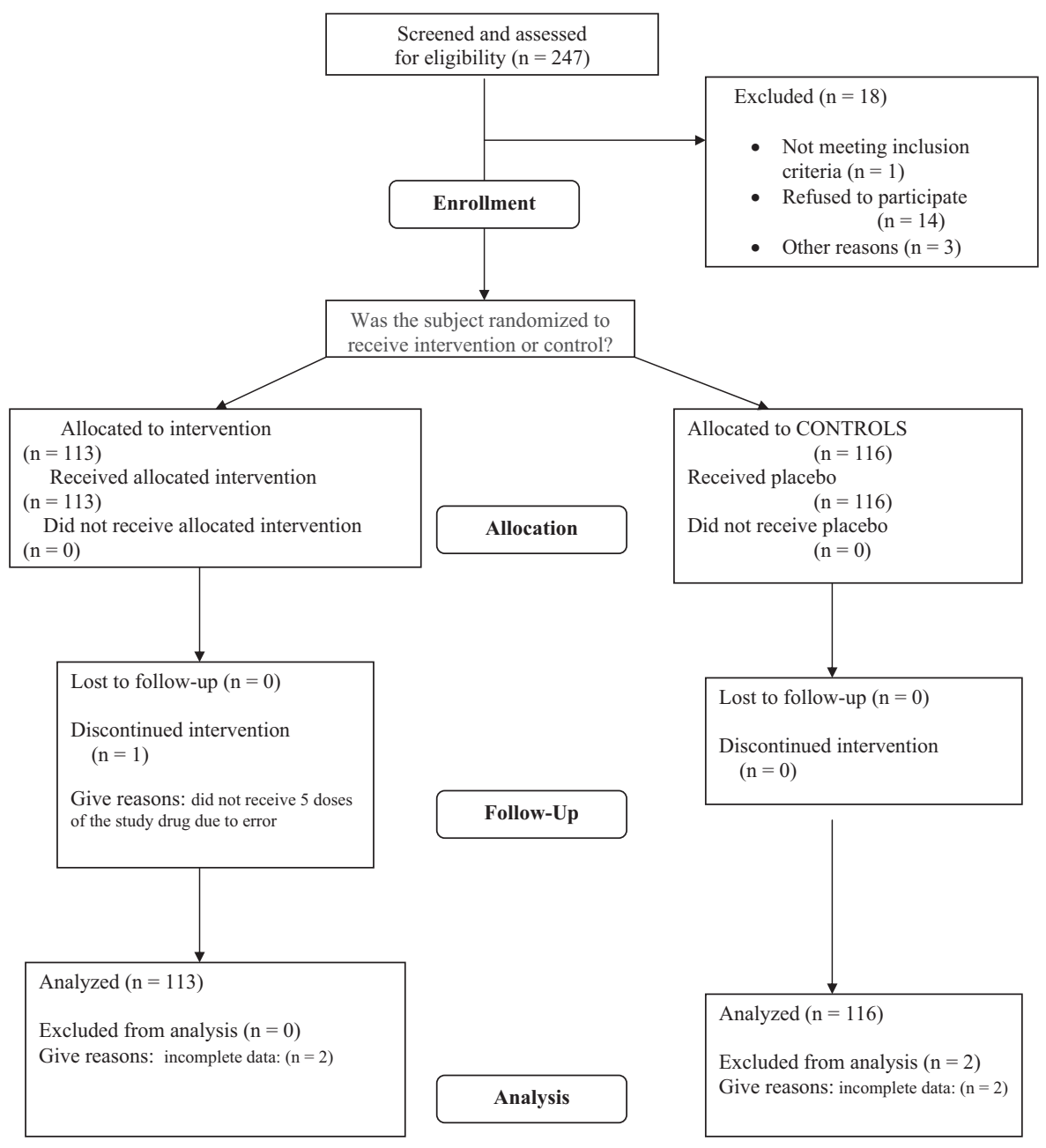

Figure 1 Flowchart (August 2005).

Table 2 Main Results

\begin{tabular}{|c|c|c|c|c|c|}
\hline & Lutein & Placebo & OR & $95 \% \mathrm{Cl}$ & $p$ Value \\
\hline \multicolumn{6}{|l|}{ Primary outcomes } \\
\hline Threshold ROP & $7 / 113(6.2 \%)$ & $12 / 116(10.3 \%)$ & 0.60 & $0.24-1.46$ & 0.18 \\
\hline NEC $>$ 2nd stage & $2 / 113(1.7 \%)$ & $6 / 116(5.1 \%)$ & 0.34 & $0.07-1.66$ & 0.15 \\
\hline BPD & $5 / 113(4.5 \%)$ & $12 / 116(10.3 \%)$ & 0.43 & $0.15-1.17$ & 0.07 \\
\hline \multicolumn{6}{|l|}{ Secondary outcomes } \\
\hline ROP all stages & $23 / 113(20.3 \%)$ & $27 / 116(23.2 \%)$ & 0.87 & $0.53-1.43$ & 0.35 \\
\hline Progression from ROP any stage to threshold ROP & $7 / 23(0.30)$ & $12 / 27(0.44)$ & 0.68 & $0.32-1.44$ & 0.23 \\
\hline Mortality (all-cause, prior to discharge) & $3.8 \%$ & $4.6 \%$ & 0.72 & $0.22-1.59$ & 0.89 \\
\hline Transfusions (mean \pm SD) & $3.1 \pm 2$ & $4.0 \pm 3$ & 0.76 & $0.20-1.49$ & 0.78 \\
\hline Hyperbilirubinemia $>14.0$ mg/dL & $6 / 113(5.3 \%)$ & $7 / 116(6.0 \%)$ & 0.85 & $0.59-1.70$ & 0.48 \\
\hline Late-onset sepsis & $17.5 \%$ & $20.3 \%$ & 0.83 & $0.42-1.67$ & 0.88 \\
\hline Severe (grade 3-4) intraventricular hemorrhage & $4.0 \%$ & $4.8 \%$ & 0.85 & $0.36-1.88$ & 0.90 \\
\hline Threshold ROP and/or BPD and/or NEC >2nd stage & $13 / 113(11.5 \%)$ & $23 / 116(19.8 \%)$ & 0.580 & $0.30-1.08$ & 0.08 \\
\hline
\end{tabular}

BPD, bronchopulmonary dysplasia; $\mathrm{Cl}$, confidence interval; NEC, necrotizing enterocolitis; OR, odds ratio; ROP, retinopathy of prematurity; SD, standard deviation. 
Table 3 Liver Function Tests Results

\begin{tabular}{|l|l|l|l|}
\hline & Group A: Lutein $(\boldsymbol{n}=113)$ & Group B: Placebo $(\boldsymbol{n}=116)$ & $p$ Value Group A versus Group B \\
\hline AST all infants & $21.3( \pm 11)$ & $24.3( \pm 10)$ & 0.80 \\
\hline ALT all infants & $18.5( \pm 13)$ & $19.9( \pm 12)$ & 0.65 \\
\hline YGT all infants & $152( \pm 78)$ & $119( \pm 65)$ & 0.08 \\
\hline Direct bilirubin all infants & $2.2( \pm 1.6)$ & $2.3( \pm 1.8)$ & 0.78 \\
\hline
\end{tabular}

All values are in mg/dL. AST, aspartate aminotransferase; ALT, alanine aminotransferase; yGT, gamma-glutamil transferase.

Our aim was to establish whether supplementation of lutein + zeaxanthin is safe and helps prevent ROP, NEC, and BPD in VLBW infants. Unfortunately, the results are inconclusive.

ROP, NEC, and BPD manifested a decreasing trend in the infants who received lutein + zeaxanthin (-Table 2 ). Supplemented infants had a $40 \%$ reduction in threshold ROP, a $52 \%$ reduction in BPD, a 73\% reduction in NEC, and a $35 \%$ reduction in the rate of progression from stage I or II ROP to threshold ROP. Although these decreases are not statistically significant, they appear relevant and are consistent among the different outcomes.

Of note, no pharmacodynamics/pharmacokinetics data are available in literature to suggest optimal dosing, and we identified the dosage to be used in this study on the basis of the GRAS declaration-related documents, ${ }^{10}$ which do not specifically refer to preterm infants. Therefore, we cannot exclude that dosing should be increased to achieve a positive effect in preterm infants whose risk for outcomes related to oxidative stress is high.

We used the same dosage of lutein + zeaxanthin supplements for all patients irrespective of the degree of prematurity and of type of feeding (maternal, donor, formula milk, or mixed). It would be interesting to understand whether these variables should affect optimal dosage.

It is also possible that, although we enrolled over 200 infants, the sample size was still too small. This limitation is inherent to the fact that this study to our knowledge is the first of its kind.

The rationale for supplementing carotenoids in premature infants is based on several points.

In the neonatal period, fresh, not-processed human milk is the main dietary source of lutein and zeaxanthin, ${ }^{20,21}$ and breast-fed infants have higher mean serum lutein concentrations than infants who consume formula unfortified with lutein. ${ }^{20,22}$ It has been calculated that four times more lutein is needed in infant formula than in human milk to achieve similar serum lutein concentrations among breast-fed and formula-fed infants. ${ }^{20}$

Carotenoids in human milk are a main player in an antioxidative network of bioactive, human milk substances that exert protective functions against oxidative stress. Damage related to oxidative stress occurring in different peripheral tissues is an etiologic moment that is common to several severe outcomes of prematurity such as ROP, NEC, and BPD. Noteworthy, breast-feeding has been associated with lower incidence rates of all of these outcomes. 6,23
Lutein and all carotenoids provide relevant in vivo antioxidative and anti-reactive oxygen species) activities through inhibition of membrane lipids' peroxidation and scavenger radical-trapping activity and via a quenching effect toward singlet and triplet oxygen. ${ }^{24-26}$ In addition, lutein and zeaxanthin ensure protection against both light-induced and metabolic oxidative damage in the retina and in other developing tissues.

In a recent pilot RCT in healthy newborns, lutein administration proved effective in increasing the levels of biological antioxidant potential by decreasing the total hydroperoxides as markers of oxidative stress. ${ }^{8}$ Plasma $\beta$-carotene concentrations have indeed been found to be lower in BPD infants, which may result in a reduction of their antioxidant protection. ${ }^{27}$ Once more, in our study, the effect of lutein + zeaxanthin on prevention of BPD appears relevant, although not statistically significant.

Clinical signs related to oxidative stress may take years and sometimes decades to become manifest. One limitation of our study is that the outcomes were assessed at discharge or at term-corrected gestational age, whichever came first. It might be worthwhile extending the evaluation of possible effects on health of early carotenoid supplementation to childhood and adulthood. In adult humans, increased dietary intake of lutein protects against the development of early atherosclerosis. ${ }^{28}$

In this study, lutein and zeaxanthin supplementation in VLBW infants appeared well tolerated and not associated with any adverse effect or putative toxicity. This is consistent with similar safety findings in adults, ${ }^{29,30}$ where in some studies much higher concentrations lutein/zeaxanthin were in fact used.

In conclusion, this RCT does not clarify whether supplementation of lutein and zeaxanthin since birth is associated with decreased incidences of several multifactorial prematurity outcomes related to oxidative stress occurring in the early ages of life. The nonsignificant decreasing trends for ROP, NEC, and BPD disclosed by our study should be confirmed in further studies with adequately powered cohorts.

\section{Acknowledgments}

We sincerely thank H. Messner, S. Cattani, G. Lista, M. Rinaldi, L. Pugni, M.G. Romeo, R. Pedicino, P. Betta, L. Renzullo, M. Quercia, F. Ferrari, E. Gallo, R. Magaldi; The Italian Task Force for the Study and Prevention of Neonatal Fungal Infections; the Italian Society of Neonatology. 
In addition to the authors, the task force includes the following persons: Amelia Di Comite, Alessandro Borghesi, Lidia Decembrino, Chryssoula Tzialla (NICU, IRCCS S. Matteo, Pavia); Giovanni Agriesti, Caterina Franco, Stefano Rizzollo, Riccardo Arisio, Giovanna Gomirato, Chiara Giovannozzi (NICU, S. Anna Hospital, Torino); Domenico Cipolla (NICU, University of Palermo); Fabio Natale (NICU, La Sapienza University, Roma); Gennaro Vetrano (NICU, Fatebenefratelli Hospital, Benevento); Elisabetta Tridapalli, Maria Grazia Capretti (NICU, University of Bologna); PierMichele Paolillo, Simonetta Picone (Neonatology Policlinico Casilino, Roma); Elio Castagnola (Infectious Disease Unit, Gaslini Hospital, Genova); Francesco Risso (Neonatology and NICU, Gaslini Hospital, Genova).

\section{Note}

Some data from this study were presented in abstract form as preliminary data at the First International Conference on Clinical Neonatology held in Torino, Italy (November 12 to 14, 2009), and at the 2011 SPR Conference in Denver, Colorado (May 1 to 4, 2011).

\section{Conflict of Interest}

The study was partially supported by unrestricted grant provided by SOOFT Italia.

\section{References}

1 Stoll BJ, Hansen NI, Bell EF, et al; Eunice Kennedy Shriver National Institute of Child Health and Human Development Neonatal Research Network. Neonatal outcomes of extremely preterm infants from the NICHD Neonatal Research Network. Pediatrics 2010;126:443-456

2 Dani C, Giannini L, Bertini G, et al. Changes of nitric oxide, carbon monoxide and oxidative stress in term infants at birth. Free Radic Res 2007;41:1358-1363

3 Buonocore G, Groenendaal F. Anti-oxidant strategies. Semin Fetal Neonatal Med 2007;12:287-295

4 Schanler RJ, Lau C, Hurst NM, Smith EO. Randomized trial of donor human milk versus preterm formula as substitutes for mothers' own milk in the feeding of extremely premature infants. Pediatrics 2005;116:400-406

5 Hylander MA, Strobino DM, Pezzullo JC, Dhanireddy R. Association of human milk feedings with a reduction in retinopathy of prematurity among very low birthweight infants. J Perinatol 2001;21:356-362

6 Lucas A, Cole TJ. Breast milk and neonatal necrotising enterocolitis. Lancet 1990;336:1519-1523

7 Lindmark-Månsson H, Akesson B. Antioxidative factors in milk. Br J Nutr 2000;84(Suppl 1):S103-S110

8 Perrone S, Longini M, Marzocchi B, et al. Effects of lutein on oxidative stress in the term newborn: a pilot study. Neonatology 2010;97:36-40

9 Sommerburg O, Meissner K, Nelle M, Lenhartz H, Leichsenring M. Carotenoid supply in breast-fed and formula-fed neonates. Eur J Pediatr 2000;159:86-90

10 GRAS Notice No. GNR 000140 issued by CFSAN/Office of Food Additive Safety. Available at: http://www.accessdata.fda.gov/ scripts/fcn/gras_notices/GRN000385.pdf. Accessed June 22, 2012

11 Bell MJ, Ternberg JL, Feigin RD, et al. Neonatal necrotizing enterocolitis. Therapeutic decisions based upon clinical staging. Ann Surg 1978;187:1-7
12 Jobe AH, Bancalari E. Bronchopulmonary dysplasia. Am J Respir Crit Care Med 2001;163:1723-1729

13 Good WV, Hardy RJ, Dobson V, et al; Early Treatment for Retinopathy of Prematurity Cooperative Group. The incidence and course of retinopathy of prematurity: findings from the early treatment for retinopathy of prematurity study. Pediatrics 2005; $116: 15-23$

14 Manzoni P, Rinaldi M, Cattani S, et al; Italian Task Force for the Study and Prevention of Neonatal Fungal Infections, Italian Society of Neonatology. Bovine lactoferrin supplementation for prevention of late-onset sepsis in very low-birth-weight neonates: a randomized trial. JAMA 2009;302:1421-1428

15 Manzoni P, Stolfi I, Pugni L, et al; Italian Task Force for the Study and Prevention of Neonatal Fungal Infections; Italian Society of Neonatology. A multicenter, randomized trial of prophylactic fluconazole in preterm neonates. N Engl J Med 2007;356:2483-2495

16 Ascioglu S, Rex JH, de Pauw B, et al; Invasive Fungal Infections Cooperative Group of the European Organization for Research and Treatment of Cancer; Mycoses Study Group of the National Institute of Allergy and Infectious Diseases. Defining opportunistic invasive fungal infections in immunocompromised patients with cancer and hematopoietic stem cell transplants: an international consensus. Clin Infect Dis 2002;34:7-14

17 Manzoni P, Pedicino R, Stolfi I, et al; Task Force per le infezioni fungine neonatali del GSIN; Socieà Italiana di Neonatologia. [Criteria for the diagnosis of systemic fungal infections in newborns: a report from the Task Force on neonatal fungal infections of the GSIN]. Pediatr Med Chir 2004;26:89-95

18 Papile LA, Burstein J, Burstein R, Koffler H. Incidence and evolution of subependymal and intraventricular hemorrhage: a study of infants with birth weights less than 1,500 gm. J Pediatr 1978;92:529-534

19 Alderson P, Green S, Higgins JPT, Eds. Cochrane Reviewers' Handbook 4.2.2. Available at: http://www.mv.helsinki.fi/home/hemila/ karlowski/handbook_4_2_2_Karlowski.pdf. Accessed June 2012

20 Bettler J, Zimmer JP, Neuringer M, DeRusso PA. Serum lutein concentrations in healthy term infants fed human milk or infant formula with lutein. Eur J Nutr 2010;49:45-51

21 Tacken KJ, Vogelsang A, van Lingen RA, Slootstra J, Dikkeschei BD, van Zoeren-Grobben D. Loss of triglycerides and carotenoids in human milk after processing. Arch Dis Child Fetal Neonatal Ed 2009;94:F447-F450

22 Capeding R, Gepanayao CP, Calimon N, et al. Lutein-fortified infant formula fed to healthy term infants: evaluation of growth effects and safety. Nutr J 2010;9:22

23 Okamoto T, Shirai M, Kokubo M, et al. Human milk reduces the risk of retinal detachment in extremely low-birthweight infants. Pediatr Int 2007;49:894-897

24 Thörne A, Aberg W, Carneheim C, Olivecrona T, Nordenström J. Influence of trauma on plasma elimination of exogenous fat and on lipoprotein lipase activity and mass. Clin Nutr 2005;24:66-74

25 Mares-Perlman JA, Millen AE, Ficek TL, Hankinson SE. The body of evidence to support a protective role for lutein and zeaxanthin in delaying chronic disease. Overview. J Nutr 2002;132:518S-524S

26 Sato J, O'Brien T, Katusic ZS, et al. Dietary antioxidants preserve endothelium dependent vasorelaxation in overfed rats. Atherosclerosis 2002;161:327-333

27 Vogelsang A, van Lingen RA, Slootstra J, et al. Antioxidant role of plasma carotenoids in bronchopulmonary dysplasia in preterm infants. Int J Vitam Nutr Res 2009;79:288-296

28 Dwyer JH, Navab M, Dwyer KM, et al. Oxygenated carotenoid lutein and progression of early atherosclerosis: the Los Angeles atherosclerosis study. Circulation 2001;103:2922-2927

29 Eye Disease Case-Control Study Group. Antioxidant status and neovascular age-related macular degeneration. Arch Ophthalmol 1993;111:104-109

30 Gale CR, Hall NF, Phillips DI, Martyn CN. Lutein and zeaxanthin status and risk of age-related macular degeneration. Invest Ophthalmol Vis Sci 2003;44:2461-2465 